\title{
The Risk and Prevalence of COVID-19 Infection in Colorectal Cancer Patients: a Systematic Review and Meta-analysis
}

\author{
Mohammad Hossein Antikchi ${ }^{1} \cdot$ Hossein Neamatzadeh ${ }^{2,3} \cdot$ Yaser Ghelmani ${ }^{4}$. Jamal Jafari-Nedooshan ${ }^{5}$. \\ Seyed Alireza Dastgheib ${ }^{6}$. Shadi Kargar ${ }^{5} \cdot$ Mahmood Noorishadkam ${ }^{2} \cdot$ Reza Bahrami $^{7}$. \\ Mohammad Hossein Jarahzadeh ${ }^{8}$
}

Accepted: 24 September 2020 / Published online: 30 September 2020

(C) Springer Science+Business Media, LLC, part of Springer Nature 2020

\begin{abstract}
Background Patients with cancer might be at an increased risk of infection with COVID-19 and a more severe disease course. However, different tumor types have differing susceptibility to the infection and COVID-19 phenotypes. Thus, the risk and prevalence of COVID-19 is not uniform across the different tumor types. Here, we performed a meta-analysis to estimate the risk and prevalence of COVID-19 infection in colorectal cancer (CRC) patients.

Methods A comprehensive literature search was performed up to July 25, 2020, thorough PubMed, Web of Science, Scopus, Google Scholar, CNKI, CBM, China Science, Wan Fang, and SciELO databases. The risk of COVID-19 infection in CRC patients was performed based on the odds ratios (ORs) and $95 \%$ confidence interval $(95 \% \mathrm{CI})$.

Results A total of six studies with 204 different cancer patients with COVID-19 and 92 CRC infected patients with COVID-19 were selected. Our results showed that the prevalence of COVID-19 infection in CRC patients was $45.1 \%$ in the global population. The pooled data showed that there is no a significant risk of infection with COVID-19 in CRC patients in the global population ( $\mathrm{OR}=0.261,95 \% \mathrm{CI} 0.099-0.533, p=0.082$ ). However, when subgroup analysis was performed based on country of origin, we found a significant correlation in Chinese CRC patients (OR $=0.221,95 \%$ CI $0.146-0.319, p \leq 0.001)$.

Conclusions This study results revealed that Chinese CRC patients harbored a higher risk of COVID-19 infection. However, more multicenter, larger sample sizes and high-quality studies are required to verify this meta-analysis result.
\end{abstract}

Keywords SARS-CoV-2 $\cdot$ COVID- $19 \cdot$ Colorectal cancer $\cdot$ Prevalence $\cdot$ Risk $\cdot$ Infection

Jamal Jafari-Nedooshan

j.jafari.yazd@gmail.com

1 Department of Internal Medicine, Yazd Branch, Islamic Azad University, Yazd, Iran

2 Mother and Newborn Health Research Center, Shahid Sadoughi University of Medical Sciences, Yazd, Iran

3 Department of Medical Genetics, Shahid Sadoughi University of Medical Sciences, Yazd, Iran

4 Clinical Research Development Center of Shahid Sadoughi Hospital, Shahid Sadoughi University of Medical Sciences, Yazd, Iran

5 Department of General Surgery, Shahid Sadoughi University of Medical Sciences, Yazd, Iran

6 Department of Medical Genetics, School of Medicine, Shiraz University of Medical Sciences, Shiraz, Iran

7 Neonatal Research Center, Shiraz University of Medical Sciences, Shiraz, Iran

8 Department of Anesthesiology and Critical Care, Shahid Sadoughi University of Medical Sciences, Yazd, Iran

\section{Introduction}

In late December 2019, the novel severe acute respiratory syndrome coronavirus 2 (SARS-CoV-2 or COVID-19) was discovered in the Chinese city of Wuhan where it quickly led to a severe outbreak globally [1,2]. The SARS-CoV-2 has caused more than 28 million cases of COVID-19 and 906,000 deaths globally [3, 4]. The COVID-19 pandemic has a big number of infected cases that far exceeded the equivalents of severe acute respiratory syndromes (SARS) and Middle East respiratory syndrome (MERS) [5]. At present, with no specific therapeutic interventions or vaccines, COVID-19 has continued to cause a human and severe economic disruption affecting millions of people [6]. The disease pandemic and mortality showed the necessity for accurate and convenient approaches to diagnose, treat, and therapeutically monitor respiratory viral infections $[7,8]$. Several efforts have combined to rapidly develop a treatment and/or a vaccine to overcome the COVID-19 [9]. 
Individuals with an underlying disease are more likely to develop severe course of COVID-19 infection [10, 11]. Moreover, they experienced high rates of anxiety, depression, distress, and insomnia in cancer patients during the COVID19 pandemic $[12,13]$. Globally, cancer care has experienced a crisis during the pandemic, because most health care systems have been required to reorganize their infrastructure and staffing to manage the pandemic $[14,15]$. Thus, there may be a longer holdback for rescheduling once the moratorium is lifted [16]. Currently there are several suggestions for various practical adaptation strategies for use during infection and recovery period [17]. Cancer patients receiving anticancer regimen have been generally believed at a higher risk of COVID-19 infection than those patients who are not receiving anticancer treatment [18]. Moreover, infected cancer patients had a significantly higher mortality rate compared with noncancer patients $[19,20]$. However, it seems that the different cancer types have differing susceptibility to the infection with COVID-19 and its phenotypes. Thus, the risk and prevalence of COVID-19 is not uniform across the different cancer types [21]. The pandemic has brought great challenges to the important elective screening and diagnostic procedures for colorectal cancer (CRC) $[16,22]$. During the pandemic, the ability of colorectal surgeons has been affected to offer care to the CRC patients globally [22, 23]. To date, some studies have been performed to evaluate the risk and prevalence of COVID-19 in CRC patients, but the results of these studies were inconsistent due to limited sample sizes. Thus, we conducted this meta-analysis to estimate the risk and prevalence of COVID-19 infection in CRC patients using all available data.

\section{Materials and Methods}

\section{Identification of Relevant Studies}

All analyses were based on previous published studies; thus, no ethical approval and patient consent are required. We searched Medline (via PubMed), Scopus, Goggle Scholar, EMBASE, Web of Sciences, Springer Link, Elsevier, Cochrane Library database, SciELO, China Biology Medical literature (CBM), Chinese Biomedical Database (CBD), China National Knowledge Infrastructure (CNKI), China Science and Technology Journal Database (VIP), and Chinese literature (Wan Fang) database for all relevant studies related to the COVID-19 infection in CRC patients up to July 25,2020 . We used the combination of the following search terms and keywords: "Severe acute Respiratory Syndrome Coronavirus 2" OR "SARS-CoV-2" OR "Coronavirus Disease 2019" OR "COVID-19" OR “ 2019 novel coronavirus" OR "2019-nCoV" OR "Human Coronavirus 2019" OR "HCoV-19" OR "hCoV-19") AND
("Colorectal Cancer" OR "CRC" OR "Bowel Cancer" OR "Colon Cancer" OR "Rectal Cancer" OR "Tumor" OR "Cancer" OR "Neoplasm" OR "Chemotherapy" OR "Immunotherapy" OR "Radiotherapy"). Moreover, the reference lists of all retrieved articles and reviews were screened for additional articles. Articles included in the meta-analysis were on human beings and published in the primary literature and had no obvious overlap of subjects with other studies. There were no restrictions based on language, sample size, type of cancer (in control group), country of origin, gender of patients, and received treatment.

\section{Inclusion and Exclusion Criteria}

The studies included in the present meta-analysis were required to meet the following criteria: (1) studies with casecontrol and cohort design; (2) studies evaluated frequency of COVID-19 infection in CRC patients; (3) only the studies with different cancer patients in the control group were considered; (4) sufficient genotype data were presented to calculate the odds ratio (OR) with $95 \%$ confidence interval (CI). In addition, the following exclusion criteria were also used: (1) unavailable raw info for extraction; (2) in vitro studies; (3) studies only involved infected CRC patients (case only studies); and (4) case report, abstracts, posters, conference papers, editorials, reviews, and meta-analyses.

\section{Data Extraction}

Data was carefully extracted by two authors independently. When the authors were not in agreement, a third author was involved to reach an agreement. The following data were extracted from each study: first author's name, year of publication, country or region, ethnic group of the study population, and total numbers of all cancer cases and CRC patients. We make an effort to guarantee that no duplicated data for the same group of cancer patients were included in the current meta-analysis. If the same population was used in multiple studies, only the most complete or latest study was selected for further analysis.

\section{Data Synthesis}

The risk of COVID-19 infection in CRC patients was assessed by odds ratios (ORs) with $95 \%$ confidence intervals (CIs). The significance of pooled ORs was determined using the $Z$-test with $p<0.05$ defined as the significance threshold. The Cochran $Q$-test was used to assess between-study heterogeneity, in which a $p$ value $<0.01$ indicates significant heterogeneity. In addition, the $I^{2}$ statistic was applied to qualify the heterogeneity ( $I^{2}$ range of 0 to $100 \%$ : $I^{2}<25 \%$, no heterogeneity; $I^{2}$ $25-50 \%$, moderate heterogeneity; $I^{2}>50 \%$, large or 
extreme heterogeneity). When significant heterogeneity was confirmed, the analysis was conducted under the random effects model (DerSimonian-Laird method). Otherwise, a fixed effects model (Mantel-Haenszel method) was employed to analyze data of the pooled ORs. Then, to identify the source of heterogeneity and test the stability of our pooled data, a sensitivity analysis was used using the leave-one-out method. The potential publication bias was tested by visual inspection of the funnel plot, in which an asymmetric plot suggests a possible publication bias. Moreover, Egger's weighted regression test was used to assess the publication bias statistically. If the publication bias tests indicated existence of potentially publication bias, the Duval and Tweedie "trim-and-fill" method was applied to adjust the results. The process of statistical analysis was conducted with the Comprehensive Meta-Analysis (CMA) software version 2.0 (Biostat, USA). Two-sided $p$ values $<0.05$ were considered statistically significant.

\section{Quality Assessment}

To test the methodological quality of the selected studies in the meta-analysis, we performed based on the NewcastleOttawa scale (NOS). The NOS ranges from zero to nine stars, and a score $\geq 6$ was considered to be of high quality. Two authors assessed the quality of included studies independently, and all disagreements were resolved by discussion.

\section{Results}

\section{Characteristics of Eligible Studies}

The study selection procedure is shown in Fig. 1. A total of 96 publications from PubMed, Goggle Scholar, EMBASE, Cochrane Library database, SciELO, CBD, CNKI, and Web of Science databases were reviewed. After the titles and abstracts were read, 28 duplicated records were excluded.
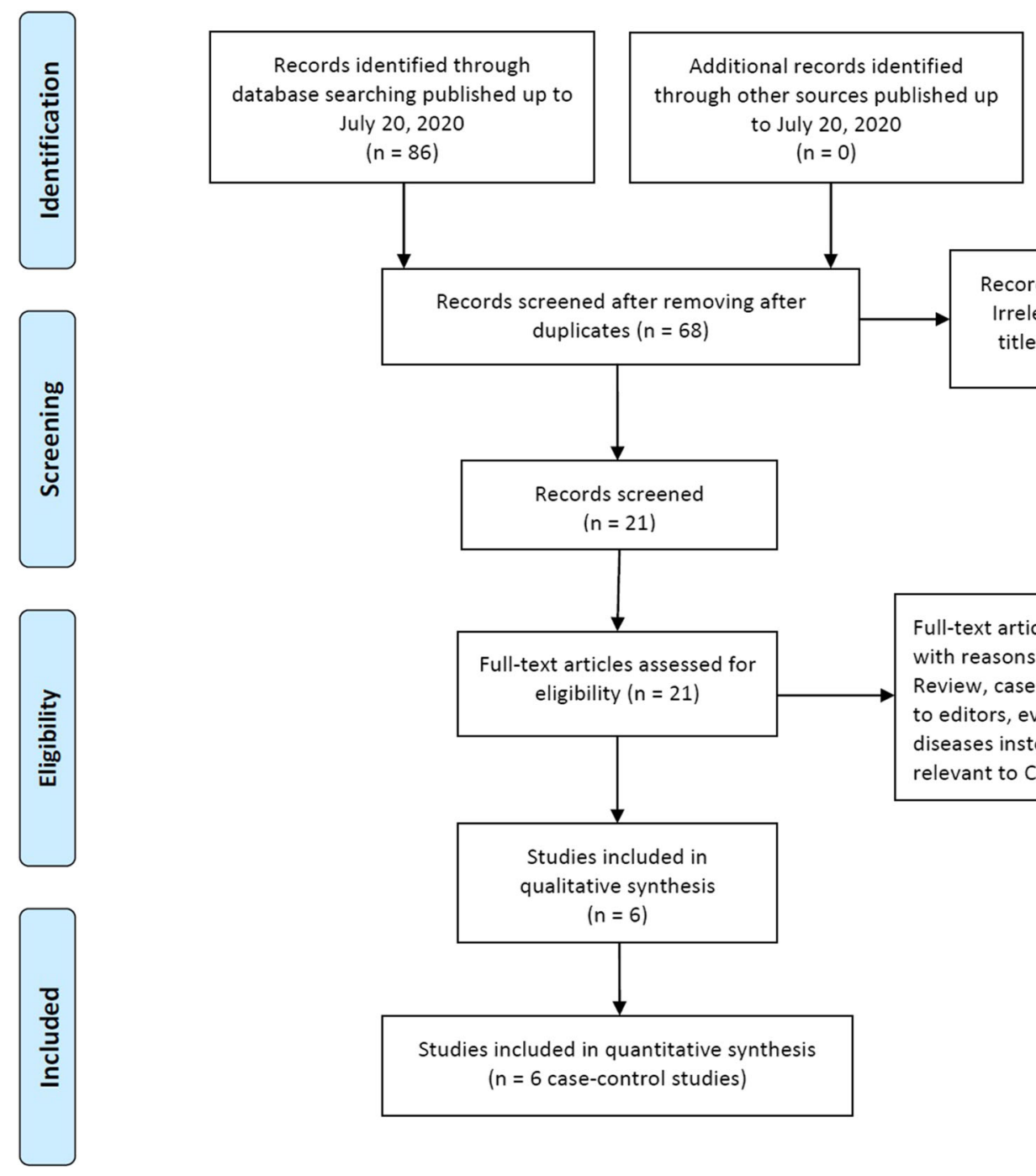

$(n=86)$
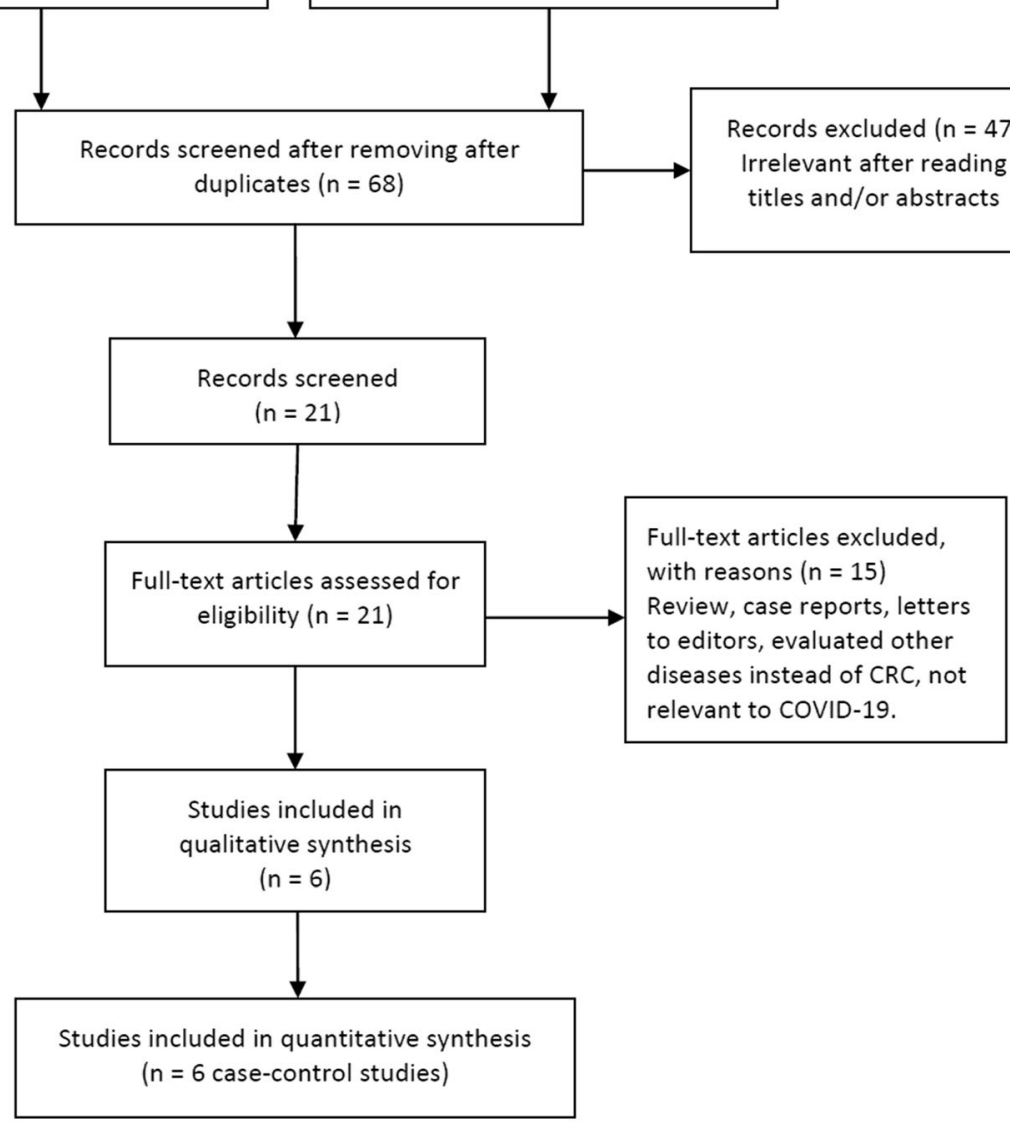

Fig. 1 Flowchart of literature search and selection process 
Table 1 Main characteristics of the included studies in the metaanalysis.

\begin{tabular}{lllll}
\hline Author/year & $\begin{array}{l}\text { Country/ } \\
\text { ethnicity }\end{array}$ & $\begin{array}{l}\text { All cancer cases } \\
\text { with COVID-19 }(n=204)\end{array}$ & $\begin{array}{l}\text { CRC cases with } \\
\text { COVID-19 }(n=92)\end{array}$ & NOS \\
\hline Yu et al., 2020 & China (Asian) & $12(5.9)$ & $2(2.2)$ & 7 \\
Liang et al., 2020 & China (Asian) & $18(8.8)$ & $4(4.3)$ & 8 \\
Ma et al., 2020 & China (Asian) & $37(18.1)$ & $11(11.9)$ & 8 \\
Zhang et al., 2020 & China (Asian) & $28(13.7)$ & $1(1.1)$ & 7 \\
Zhang et al., 2020 & China (Asian) & $5(2.5)$ & $72(78.3)$ & 6 \\
Aznab et al., 2020 & Iran (Asian) & $104(51.0)$ & 6 \\
\hline
\end{tabular}

Among the remaining 68 potentially relevant publications, 47 were considered improper after their titles and abstracts were read. Among the remaining eleven publications for full-text assessment, 15 unrelated publications were eliminated in accordance with the predetermined inclusion and exclusion criteria. Finally, a total of six studies [6, 24-28] with a 204 different cancer cases (lung cancer, colorectal cancer, breast cancer, esophagus cancer, bladder cancer, pancreatic cancer, ovarian cancer, cervical cancer, and brain tumor) and $92 \mathrm{CRC}$ patients were included in this meta-analysis. The baseline characteristics of the selected studies are presented in Table 1. All included studies were published between July 12, 2020, and December 12, 2020. Of those studies, five studies were published in English and one in Chinese. The sample size of CRC patients ranged from 5 to 104 for different cancer cases infected with COVID-19 and 1 to 72 for CRC infected patients. The selected studies were conducted among Chinese and Iranian cancer patients. As shown in Table 1, the quality scores ranged from 6 to 8, indicating that all included studies had high-quality scores.

\section{The Prevalence and Risk of COVID-19 Infection}

The pooled data on prevalence of COVID-19 infection in people with CRC are shown in Table 2. The chi-squaredbased $Q$-statistic test showed that there was a significant heterogeneity $\left(I^{2}=88.42 \%, p \leq 0.001\right)$; therefore, we applied a random effects model for the risk estimation. Our results showed that the prevalence of COVID-19 infection in CRC patients was $45.1 \%$ in global population. The pooled data showed that there is no significant correlation for COVID-19 infection in CRC patients in the global population $(\mathrm{OR}=$
0.261, 95\% CI 0.099-0.533, $p=0.082$; Fig. 2a). However, when subgroup analysis was performed based on country of origin, we found a significant correlation in Chinese CRC patients $(\mathrm{OR}=0.221,95 \%$ CI $0.146-0.319, p \leq 0.001$; Fig. 2b). The $Q$-statistic test showed that there was no significant heterogeneity $\left(I^{2}=13.31 \%, p=0.329\right)$ by the subgroup analysis. Thus, a fixed effects model was used for the risk estimation, in which it assumes that each of the studies included is estimating the same underlying parameter and the true effect size is the same in the selected studies.

\section{Publication Bias}

Begg's and Egger's linear regression tests were performed to evaluate the potential publication bias for risk of COVID-19 infection in CRC patients in the global population. The shapes of the funnel plots showed evidence of publication bias in the global population. Moreover, Egger's test found a publication bias in the global population $\left(P_{\text {Begg's }}=1.000 ; P_{\text {Egger's }}=\right.$ 0.026). Thus, the Duval and Tweedie nonparametric "trimand-fill" method was used to adjust the pooled risk for risk of COVID-19 infection in CRC patients in the global population (Fig. 3). The "trim-and-fill" method did not significantly change results, indicating that our data were statistically robust and reliable.

\section{Discussion}

Individuals with cancer had a higher risk of COVID-19 infection due to poor immunity from tumor load and malnourishment [29, 30]. Some studies demonstrated that

Table 2 Summary risk estimates for the risk of COVID-19 infection in CRC patients

\begin{tabular}{|c|c|c|c|c|c|c|c|c|c|}
\hline \multirow[t]{2}{*}{ Subgroup } & \multirow[t]{2}{*}{ Type of model } & \multicolumn{2}{|c|}{ Heterogeneity } & \multicolumn{4}{|c|}{ Odds ratio } & \multicolumn{2}{|c|}{ Publication bias } \\
\hline & & $I^{2}(\%)$ & $p_{\mathrm{H}}$ & OR & $95 \% \mathrm{CI}$ & $Z_{\text {test }}$ & $p_{\mathrm{OR}}$ & $P_{\text {Begg's }}$ & $P_{\text {Egger's }}$ \\
\hline Global population & Random & 88.42 & $\leq 0.001$ & 0.261 & $0.099-0.533$ & -1.738 & 0.082 & 1.000 & 0.026 \\
\hline Chinese population & Fixed & 13.31 & 0.329 & 0.221 & $0.146-0.319$ & -4.905 & $\leq 0.001$ & 0.462 & 0.198 \\
\hline
\end{tabular}


a

Study name

$\begin{array}{lccccc} & \begin{array}{c}\text { Event } \\ \text { rate }\end{array} & \begin{array}{c}\text { Lower } \\ \text { limit }\end{array} & \begin{array}{c}\text { Upper } \\ \text { limit }\end{array} & \text { Z-Value } & \text { p-Value } \\ \text { Yu et al., 2020 } & 0.167 & 0.042 & 0.477 & 2.078- & 0.038 \\ \text { Liang et al., 2020 } & 0.222 & 0.086 & 0.465 & 2.210- & 0.027 \\ \text { Ma et al., 2020 } & 0.297 & 0.173 & 0.461 & 2.392- & 0.017 \\ \text { Zhang et al., 2020a } & 0.071 & 0.018 & 0.245 & 3.495- & 0.000 \\ \text { Zhang et al., 2020b } & 0.200 & 0.027 & 0.691 & 1.240- & 0.215 \\ \text { Aznab et al., 2020 } & 0.692 & 0.597 & 0.773 & 3.817 & 0.000 \\ & 0.261 & 0.099 & 0.533 & 1.738- & 0.082\end{array}$

\section{Event rate and $95 \% \mathrm{Cl}$}

Relative weight

15.36

17.45

19.25

15.77

12.00

20.17

\section{b}

\section{Study name}

$\begin{array}{lccccc} & \begin{array}{c}\text { Event } \\ \text { rate }\end{array} & \begin{array}{c}\text { Lower } \\ \text { limit }\end{array} & \begin{array}{c}\text { Upper } \\ \text { limit }\end{array} & \text { Z-Value } & \text { p-Value } \\ \text { Yu et al., 2020 } & 0.167 & 0.042 & 0.477 & 2.078- & 0.038 \\ \text { Liang et al., 2020 } & 0.222 & 0.086 & 0.465 & 2.210- & 0.027 \\ \text { Ma et al., 2020 } & 0.297 & 0.173 & 0.461 & 2.392- & 0.017 \\ \text { Zhang et al., 2020a } & 0.071 & 0.018 & 0.245 & 3.495- & 0.000 \\ \text { Zhang et al., 2020b } & 0.200 & 0.027 & 0.691 & 1.240- & 0.215 \\ & 0.221 & 0.146 & 0.319 & 4.905- & 0.000\end{array}$

\section{Statistics for each study}

$-1.00$

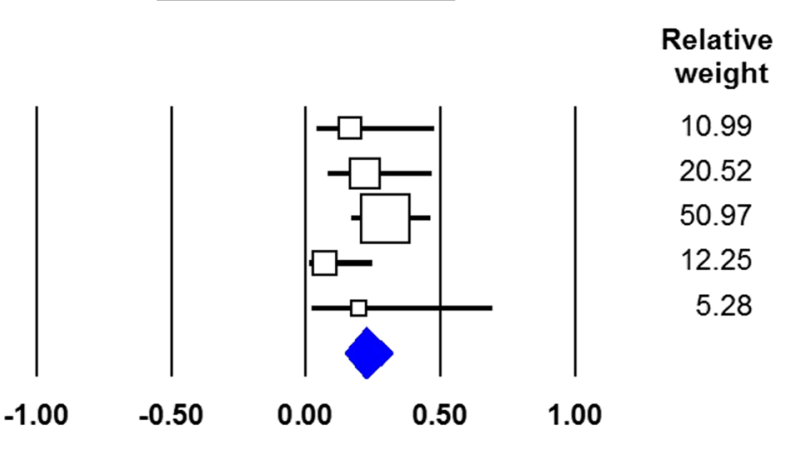

\section{Event rate and $95 \% \mathrm{Cl}$}

Fig. 2 Forest plot for risk of COVID-19 infection in CRC patients. a Global CRC patients. b Chinese CRC patients

patients with cancer seemed to be more likely to be infected with COVID-19 and have a higher risk of a severe event [31-33]. To date, some studies have been performed to evaluate the COVID-19 infection in CRC patients, but the results of these studies were inconsistent because most of these are single-center studies with limited sample sizes. Meta-analysis is a powerful and critical tool for synthesizing existing data. Thus, we performed the current meta-analysis-based six studies to estimate the risk and prevalence of COVID-19 infection in CRC patients.
Fig. 3 The funnel plots of publication bias for the risk of COVID-19 infection in CRC patients using the random model in the global population, before (blue) and after (red) the "trimand-fill" method

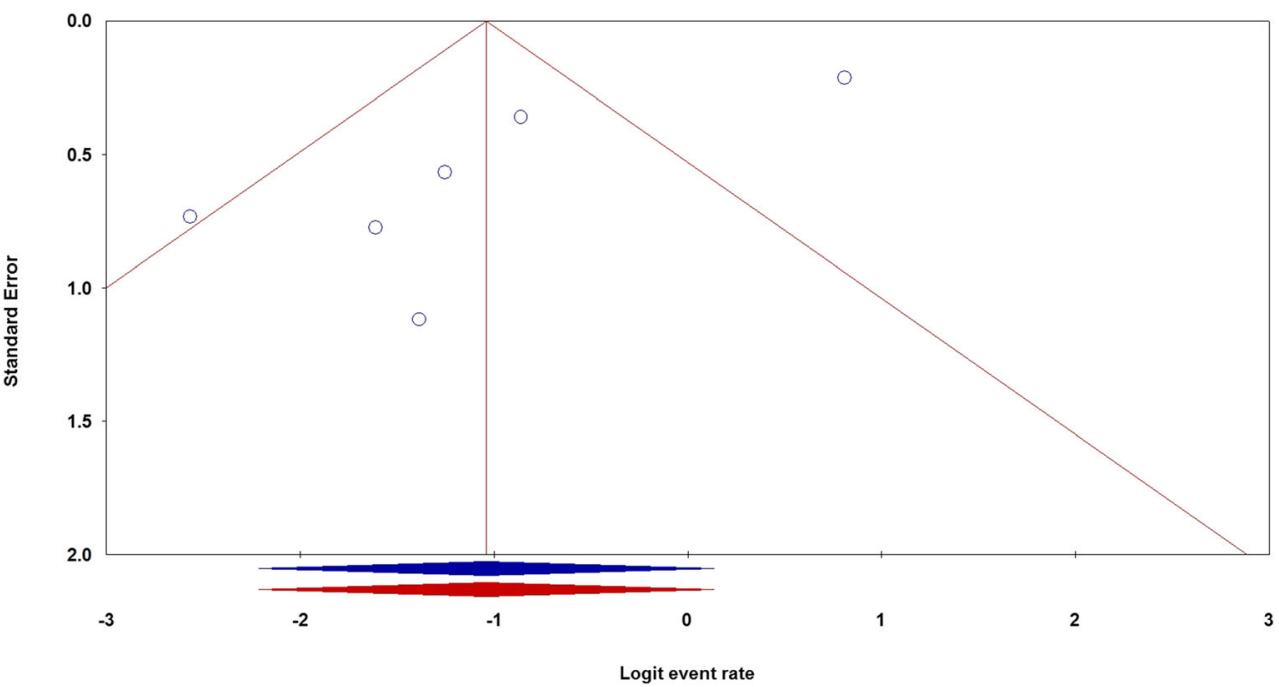


The current study showed that the prevalence of COVID-19 infection was $45.1 \%$ in CRC patients, which demonstrates a high infection with COVID-19 among those patients. Moreover, the pooled data revealed that there is no a significant correlation for COVID-19 infection in CRC patients in the global population $(\mathrm{OR}=0.261,95 \%$ CI $0.099-0.533, p=$ 0.082). However, when subgroup analysis was performed by country of origin, we found a significant correlation in Chinese CRC patients $(\mathrm{OR}=0.221,95 \%$ CI $0.146-0.319, p \leq 0.001)$. Wang et al., in a meta-analysis of six studies with 205 cancer patients, evaluated the prevalence of COVID-19 infection in different types of cancer. Their results showed that the prevalence of COVID-19 infection among CRC patients was $20.5 \%$. Moreover, their pooled data revealed that there was a significant risk for COVID-19 infection in CRC patients (95\% CI 0.13$0.29, p \leq 0.001)$ [21]. Aznab et al. in a study evaluated the prevalence of COVID-19 and its outcome in 279 cancer patients within 90 days in Kermanshah City, Iran. Their results demonstrated the COVID-19 infection in $72 \mathrm{CRC}$, eleven cases of lung cancer, five brain tumors, and 12 ovarian cancer [28].

The results of the present meta-analysis should be interpreted carefully because of the following limitations. First, the limited numbers of studies were included in the current meta-analysis, which might not provide sufficient statistical power to estimate the risk of risk of COVID-19 infection in CRC patients. Second, because we included only cancer patients infected with COVID-19, it may be caused to difficulty to weighing up with general population. Third, the included studies in the current meta-analysis were performed mostly in infected Chinese CRC patients with COVID-19, which may cause ethnicity bias. Fourth, in the current metaanalysis, only published studies in English or Chinese language were included which might cause potential selection bias. Fifth, the risk assessment in the current meta-analysis was based on unadjusted estimates, whereas other confounding factors, such as age, gender, history of other underlying conditions, treatment regime, and environmental factors, were not considered for stratified analysis due to the lack of data in the primary studies. Therefore, more precise analysis should be performed adjusted by the mentioned factors. Finally, in this study, we take into account all the available and eligible studies from multiple independent sources to evaluate the risk in CRC patients, but meta-analysis is a retrospective research which is subject to methodological limitations.

In summary, this meta-analysis result revealed that the prevalence of COVID-19 infection in CRC patients was $45.1 \%$ in global population. Pooled data showed that there was no significant risk of COVID-19 infection in CRC patients in the global population. However, subgroup analysis based on country of origin revealed a significant risk of infection among Chinese CRC patients. We strongly call for further multicenter, high-quality studies with larger sample sizes from different countries to draw more accurate results.
Acknowledgments The authors thank the editors and the anonymous reviewers for insightful suggestions on this study.

Author Contribution Mohammad Hossein Antikchi, Jamal JafariNedooshan, and Mahmood Noorishadkam are responsible as the guarantor of integrity of the entire study, study design and concepts, definition of intellectual content, and literature research. Seyed Alireza Dastgheib and Reza Bahrami are responsible for the clinical studies, experimental studies, data acquisition, and manuscript preparation. Yaser Ghelmani, Mohammad Hossein Jarahzadeh, and Hossein Neamatzadeh are responsible for the data analysis, statistical analysis, and manuscript review. All authors have read and agreed with the final version of this manuscript.

\section{Compliance with Ethical Standards}

Conflict of Interest The authors declare that they have no conflicts of interest.

\section{References}

1. Tian S, Hu N, Lou J, Chen K, Kang X, Xiang Z, et al. Characteristics of COVID-19 infection in Beijing. J Infect. 2020;80:401-6.

2. Eslami H, Jalili M. The role of environmental factors to transmission of SARS-CoV-2 (COVID-19). AMB Express. 2020;10:92.

3. Zhu FC, Guan XH, Li YH, Huang JY, Jiang T, Hou LH, et al. Immunogenicity and safety of a recombinant adenovirus type-5vectored COVID-19 vaccine in healthy adults aged 18 years or older: a randomised, double-blind, placebo-controlled, phase 2 trial. Lancet Lancet Publishing Group. 2020;396:479-88.

4. Karimi-Zarchi M, Neamatzadeh H, Dastgheib SA, Abbasi H, Mirjalili SR, Behforouz A, et al. Vertical transmission of coronavirus disease 19 (COVID-19) from infected pregnant mothers to neonates: a review. Fetal Pediatr Pathol. 2020;39:246-50.

5. Zhu H, Wei L, Niu P. The novel coronavirus outbreak in Wuhan, China. Global Health Research and Policy. Springer Science and Business Media LLC; 2020;5.

6. Zhang L, Zhu F, Xie L, Wang C, Wang J, Chen R, et al. Clinical characteristics of COVID-19-infected cancer patients: a retrospective case study in three hospitals within Wuhan, China. Ann Oncol. 2020;31:894-901.

7. Waghmare A, Krantz EM, Baral S, Vasquez E, Loeffelholz T, Chung EL, et al. Reliability of self-sampling for accurate assessment of respiratory virus viral and immunologic kinetics. J Infect Dis. 2020;jiaa451.

8. Mathuria JP, Yadav R, Rajkumar. Laboratory diagnosis of SARSCoV-2 - a review of current methods. J Infect Public Health. 2020;13:901.

9. Bahmad HF, Abou-Kheir W. Crosstalk between COVID-19 and prostate cancer. Prostate Cancer Prostatic Dis. 2020:1-3.

10. Wang B, Li R, Lu Z, Huang Y. Does comorbidity increase the risk of patients with COVID-19: evidence from meta-analysis. Aging (Albany NY). 2020;12:6049-57.

11. Hussain A, Bhowmik B. do Vale Moreira NC. COVID-19 and diabetes: knowledge in progress. Diabetes Res Clin Pract. 2020;162:108142.

12. Juanjuan L, Santa-Maria CA, Hongfang F, Lingcheng W, Pengcheng Z, Yuangbing X, et al. Patient reported outcomes of breast cancer patients during the COVID-19 outbreak in the epicenter of China: a cross sectional survey study. Clin Breast Cancer. 2020;S1526-8209(20):30147-6.

13. Ghosh J, Ganguly S, Mondal D, Pandey P, Dabkara D, Biswas B. Perspective of oncology patients during COVID-19 pandemic: a 
prospective observational study from India. JCO Global Oncol. 2020;6:844-51.

14. Tsamakis K, Gavriatopoulou M, Schizas D, Stravodimou A, Mougkou A, Tsiptsios D, et al. Oncology during the COVID-19 pandemic: challenges, dilemmas and the psychosocial impact on cancer patients. Oncol Lett. 2020;20:441-7.

15. Calabrò L, Peters S, Soria JC, Di Giacomo AM, Barlesi F, Covre A, et al. Challenges in lung cancer therapy during the COVID-19 pandemic. Lancet Respir Med. 2020;8:542-4.

16. Dockter AG, Angelos GC. Molecular-based alternatives for colorectal cancer screening during the COVID-19 pandemic. Surg Technol Int. 2020;36:143-7.

17. Nunoo-Mensah JW, Rizk M, Caushaj PF, Giordano P, Fortunato R, Dulskas A, et al. COVID-19 and the global impact on colorectal practice and surgery. Clin Colorectal Cancer. 2020;S15330028(20):30084-0.

18. Lee LYW, Cazier JB, Starkey T, Turnbull CD, Kerr R, Middleton G. COVID-19 mortality in patients with cancer on chemotherapy or other anticancer treatments: a prospective cohort study. Lancet Lancet Publishing Group. 2020;395:1919-26.

19. Sanchez-Pina JM, Rodríguez Rodriguez M, Castro Quismondo N, Gil Manso R, Colmenares R, Gil Alos D, et al. Clinical course and risk factors for mortality from COVID-19 in patients with haematological malignancies. Eur J Haematol. 2020; ejh.13493.

20. Shoumariyeh K, Biavasco F, Ihorst G, Rieg S, Nieters A, Kern W V., et al. Covid-19 in patients with hematological and solid cancers at a comprehensive cancer center in Germany. Cancer Medicine. John Wiley \& Sons, Ltd;

21. Wang B, Huang Y. Which type of cancer patients are more susceptible to the SARS-COX-2: evidence from a meta-analysis and bioinformatics analysis. Crit Rev Oncol Hematol. 2020;153:103032.

22. Ren X, Chen B, Hong Y, Liu W, Jiang Q, Yang J, et al. The challenges in colorectal cancer management during COVID-19 epidemic. Ann Transl Med. 2020;8:498.

23. Vecchione L, Stintzing S, Pentheroudakis G, Douillard JY, Lordick F. ESMO management and treatment adapted recommendations in the COVID-19 era: colorectal cancer. ESMO Open: BMJ Publishing Group; 2020.
24. Yu J, Ouyang W, Chua MLK, Xie C. SARS-CoV-2 transmission in patients with cancer at a tertiary care hospital in Wuhan, China. JAMA Oncology. 2020;6:1108-10.

25. Liang W, Guan W, Chen R, Wang W, Li J, Xu K, et al. Cancer patients in SARS-CoV-2 infection: a nationwide analysis in China. Lancet Oncol. 2020;21:335-7.

26. Ma J, Yin J, Qian Y, Wu Y. Clinical characteristics and prognosis in cancer patients with COVID-19: a single center's retrospective study. J Infect. 2020;81:318-56.

27. Zhang Y, Li M, Gan L, Li B. Analysis of clinical characteristics of 5 tumor patients with coronavirus disease 2019. Guangdong Med J. 2020;41.

28. Aznab M. Evaluation of COVID 19 infection in 279 cancer patients treated during a 90-day period in 2020 pandemic. Int J Clin Oncol. 2020;25:1581-6.

29. Dai M, Liu D, Liu M, Zhou F, Li G, Chen Z, et al. Patients with cancer appear more vulnerable to SARS-CoV-2: a multicenter study during the COVID-19 outbreak. Cancer Discov. 2020;10: 783.

30. Yang F, Shi S, Zhu J, Shi J, Dai K, Chen X. Analysis of 92 deceased patients with COVID-19. J Med Virol. 2020. https://doi.org/10. 1002/jmv.25891.

31. Moujaess E, Kourie HR, Ghosn M. Cancer patients and research during COVID-19 pandemic: a systematic review of current evidence. Crit Rev Oncol Hematol. 2020;150:102972.

32. Bernhardt D, Wick W, Weiss SE, Sahgal A, Lo SS, Suh JH, et al. Neuro-oncology management during the COVID-19 pandemic with a focus on WHO grade III and IV gliomas. Neuro-Oncology. 2020;22:928-35.

33. Saini KS. de las Heras B, de Castro J, Venkitaraman R, Poelman M, Srinivasan G, et al. Effect of the COVID-19 pandemic on cancer treatment and research. Lancet Haematol. 2020;7:e432-5.

Publisher's Note Springer Nature remains neutral with regard to jurisdictional claims in published maps and institutional affiliations. 